\title{
Obscured Asymptotic Giant Branch stars in the Magellanic Clouds
}

\author{
Jacco Th. van Loon \\ Astronomical Institute, University of Amsterdam, Kruislaan 403, \\ NL-1098 SJ Amsterdam, The Netherlands
}

\begin{abstract}
We report on some recent advances in the study and understanding of heavily obscured AGB stars in the Magellanic Clouds.
\end{abstract}

\section{Introduction}

Asymptotic Giant Branch (AGB) stars develop strong mass loss at rates up to $\dot{M} \sim 10^{-5} \mathrm{M}_{\odot} \mathrm{yr}^{-1}$ or more. The optical light of AGB stars with the highest $\dot{M}$ is almost entirely absorbed by their dusty circumstellar envelopes (CSEs), but re-emitted at longer wavelengths. They become very bright infrared (IR) objects, outshining any other star in a galaxy except for a few red supergiants (RSGs). During a brief period in their lives, they loose 50 to $80 \%$ of their initial mass. This makes them important contributors to the chemical enrichment of the interstellar medium. Obscured AGB stars are the main sources of dust particles in the Universe. Dust is a necessary condition for the formation of planets that may support life. Obscured AGB stars contain masers that emit at radio wavelengths, where a high velocity resolution is obtained $\left(\sim 1 \mathrm{~km} \mathrm{~s}^{-1}\right)$. Maser stars can thus be used to trace the gravitational potential in a galaxy.

Some questions remain to be answered: (1) How does an AGB star drive its mass loss, and what is the rôle of stellar pulsation in this matter? (2) How does an AGB star form a Planetary Nebula (PN), for which an enhancement of the already high $\dot{M}$ is required? (3) What is the most massive progenitor of an AGB star? (4) Which AGB stars become carbon stars, thereby completely changing the chemistry of molecule and dust formation in the CSE? (5) How do complex molecules and dust particles form in the CSE of an AGB star?

\section{The search for obscured AGB stars in the Magellanic Clouds}

The Large and Small Magellanic Clouds (LMC \& SMC) have two major advantages over the Milky Way when it comes to studying AGB stars: their distances are well determined ( $\sim 50$ and $60 \mathrm{kpc}$ ) and interstellar extinction is relatively small both in front of the MCs and within. Furthermore, the average metallicities of the intermediate age populations in the LMC and SMC are factors of respectively 2 and 5 lower than in the Milky Way. Thus, the aforementioned astrophysical problems may be investigated on their metallicity dependence.

Previous studies of AGB stars in the MCs were based on optically visible stars, with only very few obscured objects identified. Nothing was known about 


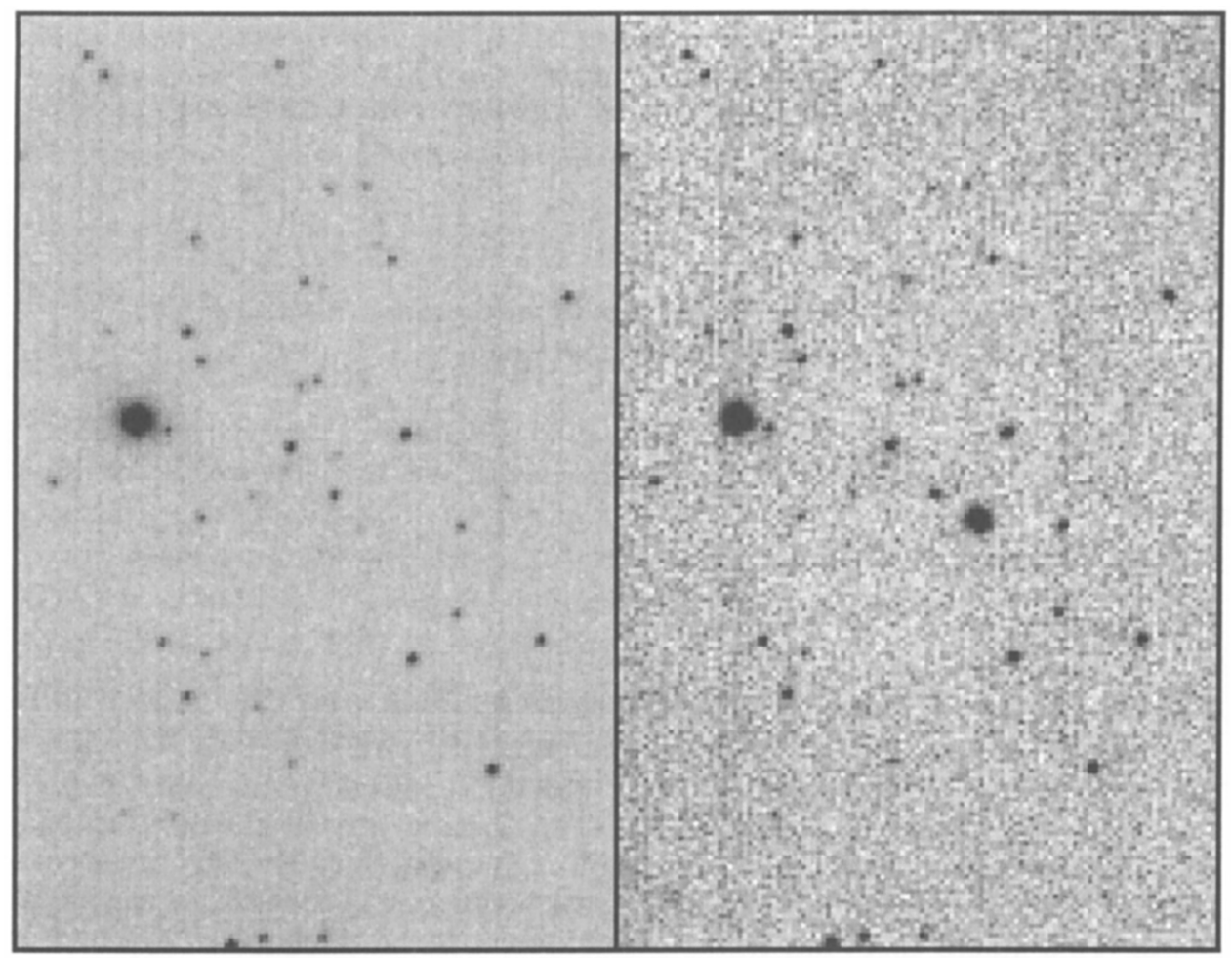

Figure 1. Identifying a magellanic AGB star as counterpart of an IRAS source. Invisible in the J-band image (at left), it rivals the brightest star in the K-band image (at right). The images were obtained using the IRAC2b near-IR camera at the ESO/MPI $2.2 \mathrm{~m}$ telescope at ESO La Silla, Chile. South is up, and East is to the right. The frames shown here measure one arcminute along the short $(\mathrm{E}-\mathrm{W})$ axis.

how AGB stars continue to evolve after they become obscured. As an example, a long standing problem has been the observation that no carbon stars in the MCs were found at luminosities higher than $2 \times 10^{4} \mathrm{~L}_{\odot}$ (Iben 1981; Hughes \& Wood 1990; Costa \& Frogel 1996). The burning of carbon into nitrogen and oxygen at the bottom of the convective mantle, Hot Bottom Burning (HBB), was proposed as a mechanism to prevent the formation of more luminous carbon stars. Alternative explanations include luminous carbon stars being dust-enshrouded. There was a clear need for a systematic (IR) search for obscured AGB stars in the MCs, and the determination of their luminosities and chemical types.

The first attempts to find obscured AGB stars in the MCs had resulted in a sample of evolved stars, comprising mainly RSGs and only a few AGB stars (Whitelock et al. 1989; Reid 1991; Wood et al. 1992). We successfully searched for counterparts of IRAS point sources in the direction of the LMC, mainly using near-IR imagery at La Silla (Loup et al. 1997; Zijlstra et al. 1996; van Loon et al. 1997, 1998a). We identified most of the counterparts as obscured AGB stars, 
all members of the LMC. As an example, we show J and K-band images of IRAS 06025-6712 obtained with the IRAC2b camera on the $2.2 \mathrm{~m} \mathrm{ESO/MPI}$ telescope at ESO La Silla, Chile (Fig. 1). Invisible in the J-band image down to $18^{\text {th }}$ magnitude, it is very bright in the $\mathrm{K}$-band image at $K=11.52 \mathrm{mag}$., and probably a carbon star. It is located in a small, anonymous open cluster. We obtained deep optical images to determine the age of the cluster, and hence the main-sequence mass of the progenitor of the obscured AGB star. We are doing the same for the luminous OH/IR star IRAS 05298-6957 in the double cluster HS327, that also contains a carbon star (van Loon et al. 1998a).

We increased the number of known obscured AGB stars in the LMC from only a few previously known objects to a sample of $\sim 50$ (van Loon et al. 1997, 1998a). In the SMC, $\sim 25$ such stars are now known (see also Groenewegen \& Blommaert 1998). The K-band distribution function of IRAS-detected AGB stars is truncated at the faint end by detection limits in the near-IR $(K \sim 19$ mag.). Indeed, for some IRAS sources we failed to identify a red stellar counterpart in the near-IR. Furthermore, there are $\sim 3$ times as many IRAS sources left unexplored, and we predicted that ISO would detect more obscured AGB stars too faint for IRAS (see also the contribution of Loup et al. in these proceedings). A large population of obscured AGB stars may explain the apparent lack of AGB stars as compared to the number of PNe in the LMC (Frogel et al. 1990; Reid et al. 1990; Wood et al. 1992). In the process, we also found post-AGB star candidates in the LMC, and highly reddened background galaxies.

\section{Chemical compositions}

We established the chemical composition for many obscured AGB stars in the MCs, using IR colours and IR spectroscopy from the ground (ESO, SAAO, CTIO) and space (IRAS, ISO). In Fig. 2 we show an example of L-band spectra obtained with the IRS at the $4 \mathrm{~m}$ Blanco telescope at Cerro Tololo, Chile, of the LMC carbon star IRAS 04496-6958 and M10 star IRAS 04509-6922 (van Loon et al. 1998a). $\mathrm{HCN}$ and $\mathrm{C}_{2} \mathrm{H}_{2}$ molecules cause strong absorption between 3.0 and $3.1 \mu \mathrm{m}$ in carbon stars, whereas oxygen-rich $(\mathrm{M})$ stars show a featureless continuum. With the VLT at ESO Paranal, Chile, it will be possible to determine in this way the chemical type of all obscured AGB stars in the MCs, and the brightest in other nearby dwarf galaxies. We also used ISO to obtain PHOT-S spectra between 2.5 and $12 \mu \mathrm{m}$ of 15 objects, and N-band spectra with ISOCAM and a Circular Variable Filter of 12 objects.

Many (but not all) obscured AGB stars in the LMC are carbon-rich. Among these, we discovered the most luminous carbon star in the MCs, IRAS 04496-6958 having a luminosity of $4 \times 10^{4} \mathrm{~L}_{\odot}$ (van Loon et al. 1998a). Thus we showed that carbon stars do exist up to the highest AGB luminosities. This inspired stellar evolution calculators to propose an explanation (Frost et al. 1998; Marigo et al. 1998): due to mass loss the stellar mantle is reduced, thereby switching off HBB that otherwise prevents massive stars from becoming carbon stars. We conclude that the population of optically visible AGB stars shows rather unevolved intermediate-age stars: they still have to pass through the phase where they will shed most of their mass and become obscured. Evolution of the chemi- 


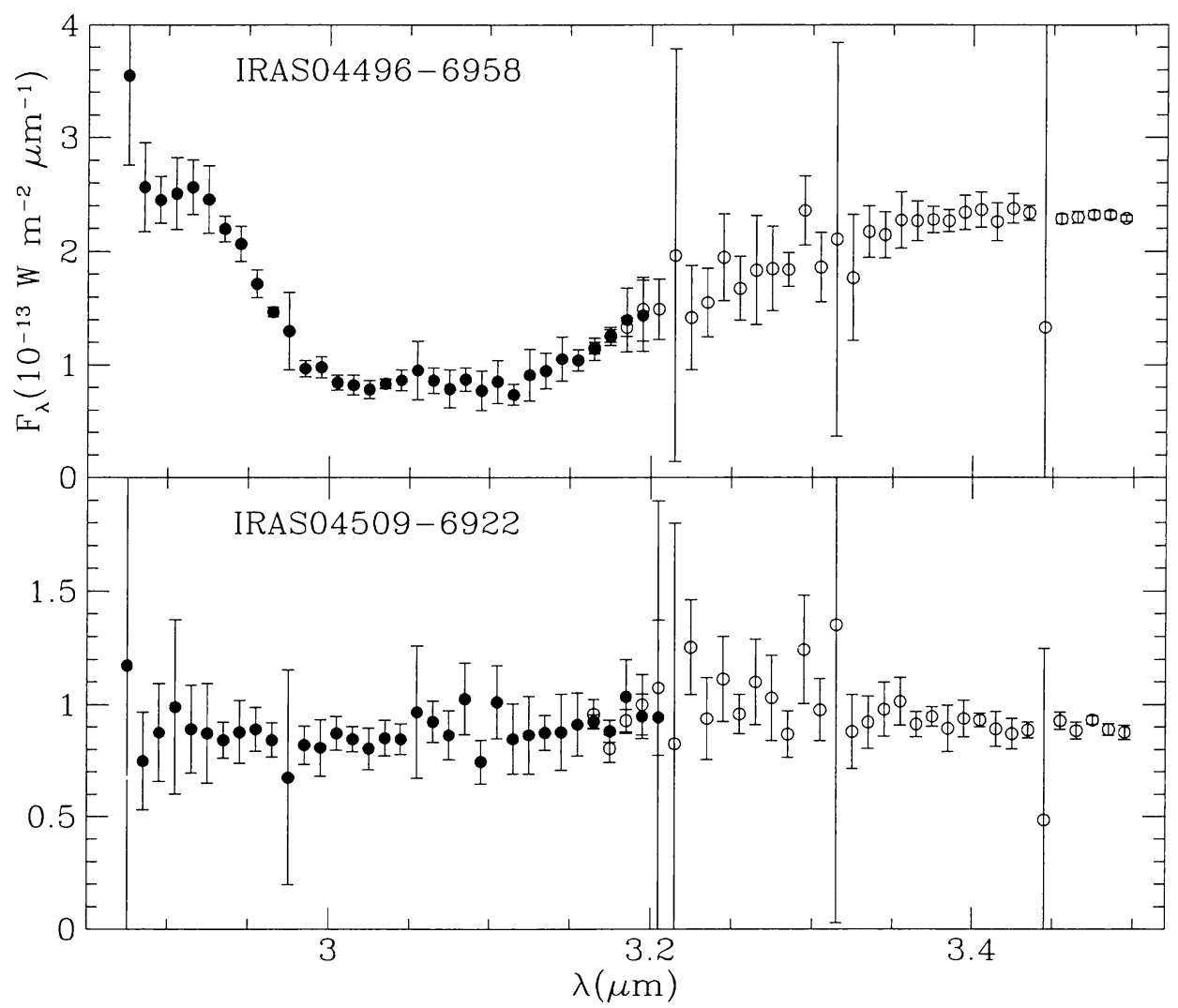

Figure 2. L-band spectra can be used to distinguish between carbon stars (IRAS 04496-6958 at top) and oxygen-rich $M$ stars (IRAS 04509-6922 at bottom) because carbon stars show strong absorption between 3.0 and $3.1 \mu \mathrm{m}$ due to $\mathrm{HCN}$ and $\mathrm{C}_{2} \mathrm{H}_{2}$ molecules. These spectra were obtained with the IRS at the $4 \mathrm{~m}$ Blanco telescope at CTIO, Chile.

cal composition of the stellar photospheres and the circumstellar dust continues during the obscuration phase.

\section{Metallicity dependence of mass-loss}

AGB mass-loss is often assumed to decrease with lower metallicity, but this still awaits strong observational support. We noticed that very obscured AGB stars exist in both the LMC and the SMC, despite their low metallicities. Assuming a dust-to-gas ratio proportional to the metallicity, and accounting for the luminosity dependence of the dust formation radius, the near-IR colours suggest that AGB stars in the Milky Way, LMC, and SMC reach similar maximum $M$. However, the optical depths of magellanic CSEs may also be large if the expansion velocity $v_{\exp }$ of the dust decreases with lower metallicity. 


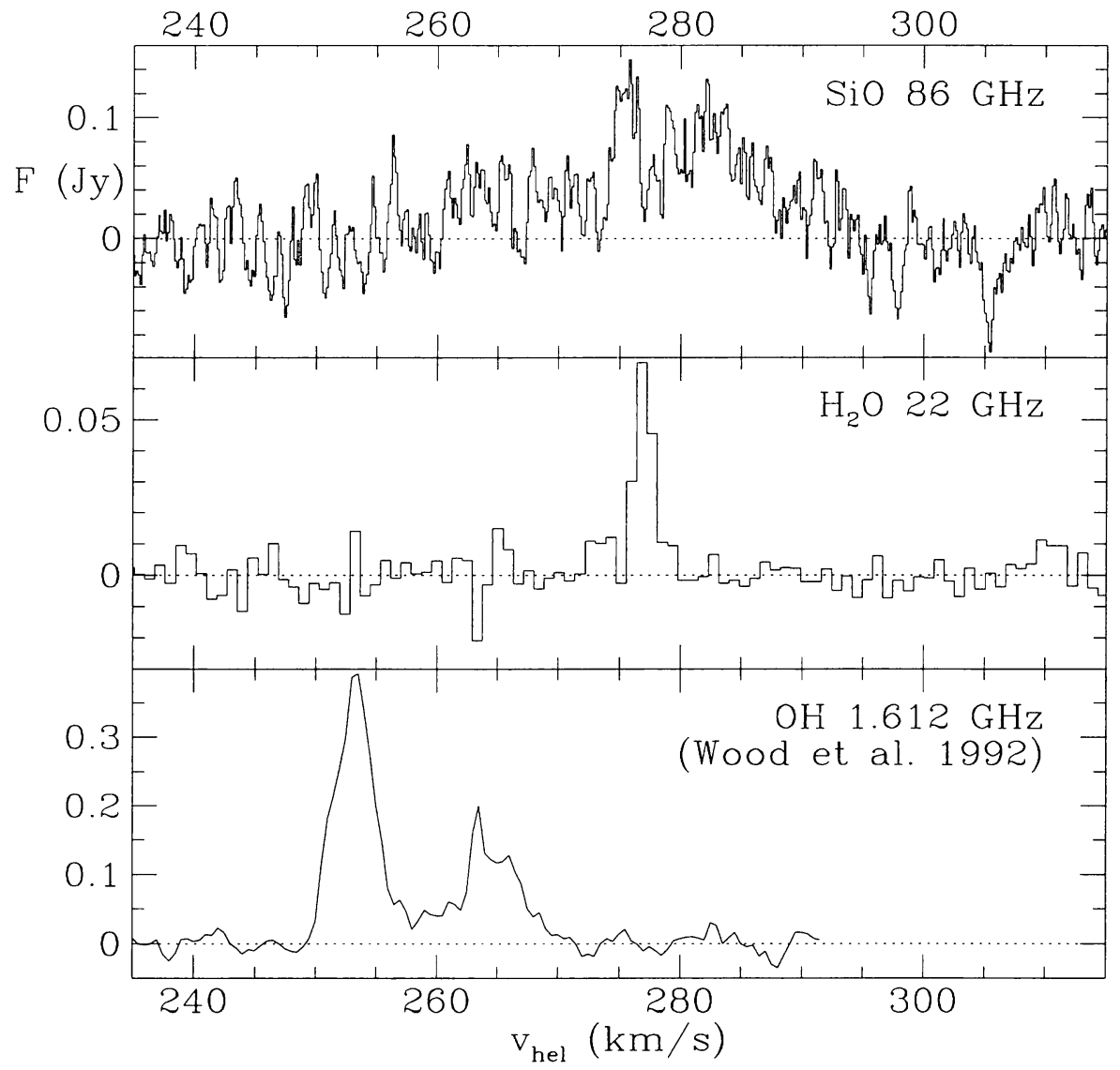

Figure 3. Maser emission can be detected from extra-galactic circumstellar envelopes. The LMC red supergiant IRAS 04553-6825 is the first star to have been detected in $\mathrm{OH}$ (Wood et al. 1986), $\mathrm{SiO}$ (van Loon et al. 1996), and $\mathrm{H}_{2} \mathrm{O}$ (van Loon et al. 1998b).

We can measure $v_{\text {exp }}$ of the gas component from circumstellar maser emission profiles. Wood et al. $(1986,1992)$ detected the first magellanic OH masers, that suggest $v_{\exp }$ to be proportional to metallicity. We have searched for more $\mathrm{OH}$ masers (van Loon et al. 1998a), and also for $\mathrm{SiO}$ and $\mathrm{H}_{2} \mathrm{O}$ masers in the LMC. We used the 15m SEST at La Silla, Chile, to detect the first extra-galactic SiO maser (van Loon et al. 1996), and we predicted and subsequently confirmed the first extra-galactic circumstellar $\mathrm{H}_{2} \mathrm{O}$ maser using the $64 \mathrm{~m}$ telescope at Parkes, Australia (van Loon 1998; van Loon et al. 1998b). We show the maser spectra of this LMC supergiant (IRAS 04553-6825) to illustrate that it has become feasible to study masers in extra-galactic CSEs (Fig. 3). We argue that $v_{\exp }$ of magellanic CSEs may have been generally underestimated. More maser searches need to be performed in the MCs before definitive conclusions can be drawn on the metallicity dependence of $v_{\exp }$ and $\dot{M}$. 


\section{Mass-loss evolution of AGB stars}

We show that AGB mass-loss does not evolve gradually, but undergoes a phase transition. In the early phase, an AGB star has a short pulsation period $(\mathrm{P}<$ 400 days) and small bolometric amplitude $\left(\Delta M_{\text {bol }}\right)$. Containing little dust, the CSE is optically thin and expands slowly $\left(v_{\exp } \sim\right.$ few $\left.\mathrm{km} \mathrm{s}^{-1}\right)$, possibly driven by radiation pressure on molecules. In the late phase, the AGB star has a long pulsation period ( $\mathrm{P}>600$ days) and large $\Delta M_{\text {bol }}$. The dusty CSE is optically thick and expands with $v_{\exp } \sim 10$ to $20 \mathrm{~km} \mathrm{~s}^{-1}$, efficiently driven by radiation pressure on dust. The mass-loss characteristics change suddenly when the star evolves from the early into the late phase, possibly due to the sensitivity of dust formation on the conditions in the CSE (e.g. Arndt et al. 1997).

Energy is stored and released during a stellar pulsation cycle. Part of the energy is thought to be used in initiating stellar mass loss. We argue from first principles that this concept naturally results in two observable facts: (1) a minimum $\Delta M_{\text {bol }}$ is needed to create a dusty CSE and high $\dot{M}$, and (2) supergiants need smaller $\Delta M_{\text {bol }}$ than AGB stars to support the same $\dot{M}$.

Acknowledgments. We thank the organisers for a pleasant and interesting conference, and for giving me the opportunity to present my work. Milhões de beijinhos para o anjinho Joana.

\section{References}

Arndt T.U., et al., 1997, A\&A 327, 614

Costa E., Frogel J.A., 1996, AJ 112, 2607

Frogel J.A., et al., 1990, ApJ 352, 96

Frost C.A., et al., 1998, A\&A 332, L17

Groenewegen M.A.T., Blommaert J.A.D.L., 1998, A\&A 332, 25

Hughes S.M.G., Wood P.R., 1990, AJ 99, 784

Iben I., Jr., 1981, ApJ 246, 278

Loup C., et al., 1997, A\&AS 125, 419

Marigo P., et al., 1998, A\&A 331, 564

Reid I.N., 1991, ApJ 382, 143

Reid I.N., et al., 1990, ApJ 348, 98

Van Loon J.Th., 1998, in "ISO's View on Stellar Evolution", Ap\&SS 255, p. 405

Van Loon J.Th., et al., 1996, A\&A 306, L29

Van Loon J.Th., et al., 1997, A\&A 325, 585

Van Loon J.Th., et al., 1998a, A\&A 329, 169

Van Loon J.Th., et al., 1998b, A\&A 337, 141

Whitelock P.A., et al., 1989, MNRAS 238, 769

Wood P.R., et al., 1986, ApJ 306, L81

Wood P.R., et al., 1992, ApJ 397, 552

Zijlstra A.A., et al., 1996, MNRAS 279, 32 